\title{
The centers of spin symmetric group algebras and Catalan numbers
}

\author{
Jill Tysse · Weiqiang Wang
}

Received: 7 November 2007 / Accepted: 13 February 2008 / Published online: 23 February 2008

(C) Springer Science+Business Media, LLC 2008

\begin{abstract}
Generalizing the work of Farahat-Higman on symmetric groups, we describe the structures of the even centers $\mathcal{Z}_{n}$ of integral spin symmetric group superalgebras, which lead to universal algebras termed as the spin FH-algebras. A connection between the odd Jucys-Murphy elements and the Catalan numbers is developed and then used to determine the algebra generators of the spin $\mathrm{FH}$-algebras and of the even centers $\mathcal{Z}_{n}$.
\end{abstract}

Keywords Spin symmetric groups · Jucys-Murphy elements · Catalan numbers

\section{Introduction}

1.1

In a fundamental paper [2], Farahat and Higman discovered some remarkable multiplicative structures of the centers of the integral group algebras for the symmetric groups $S_{n}$ with respect to the basis of conjugacy class sums. This led to two universal algebras, $K$ and $G$, which were then shown to be polynomial algebras with a distinguished set of ring generators. As an immediate consequence, the center of the integral group algebra for $S_{n}$ is shown in [2] to have the first $n$ elementary symmetric polynomials of the Jucys-Murphy elements as its ring generators (which is a modern reinterpretation since the papers [6,9] appeared after [2]), and this has implications on modular representations of the symmetric groups. A symmetric function interpretation of the class sum basis for $G$ was subsequently obtained by Macdonald [8,

J. Tysse · W. Wang (凶)

Department of Mathematics, University of Virginia, Charlottesville, VA 22904, USA

e-mail:ww9c@ virginia.edu

J. Tysse

e-mail: jem4t@virginia.edu 
pp.131-4]. Some results of Farahat-Higman were generalized to the wreath products by the second author and they admit deep connections with the cohomology rings of Hilbert schemes of points on the affine plane or on the minimal resolutions (see Wang $[14,15])$.

I. Schur in a 1911 paper [12] initiated the spin representation theory of the symmetric groups by first showing that $S_{n}$ admits double covers $\widetilde{S}_{n}$, nontrivial for $n \geq 4$ :

$$
1 \longrightarrow\{1, z\} \longrightarrow \widetilde{S}_{n} \longrightarrow S_{n} \longrightarrow 1
$$

where $z$ is a central element of $\widetilde{S}_{n}$ of order 2. We refer to Józefiak [4] (also [5]) for an excellent modern exposition of Schur's paper via a systematic use of superalgebras. Given a commutative ring $R$ with no 2-torsion, we form the spin symmetric group algebra $R S_{n}^{-}=R \widetilde{S}_{n} /\langle z+1\rangle$, which has a natural superalgebra structure.

\section{2}

The goal of this paper is to formulate and establish the spin group analogue of the fundamental work of Farahat-Higman. It turns out that all the results of FarahatHigman [2] afford natural spin generalizations but the combinatorial aspect becomes much richer and more involved.

It is known (cf. [4]) that the even center $\mathcal{Z}_{n}$ of the superalgebra $R S_{n}^{-}$has a basis given by the even split class sums of $\widetilde{S}_{n}$. We first show that the structure constants in $\mathcal{Z}_{n}$ with respect to the class sum basis are polynomials in $n$ and describe a sufficient condition for the independence of $n$ of these structure constants, just as in [2]. This leads to two universal algebras, $\mathcal{K}$ and $\mathcal{F}$, which we call the filtered and graded spin FH-algebras respectively. The proofs here are similar to the ones in [2], but we need to carefully keep track of the (sometimes subtle) signs appearing in the multiplications of cycles in $R S_{n}^{-}$. Our treatment systematically uses the notion of modified cycle type (cf. [8, p.131]).

The odd Jucys-Murphy elements $M_{i}$ for $R S_{n}^{-}$introduced by Sergeev [13] (also cf. related constructions by Nazarov [11]) will play the role of the usual Jucys-Murphy elements for $S_{n}$. The odd Jucys-Murphy elements anti-commute with each other, and a conceptual framework has been provided by the notion of degenerate spin affine Hecke algebras (see Wang [16]). We show that the top degree term of any elementary symmetric function in the squares $M_{i}^{2}$ is a linear combination of the even split class sums with coefficients given explicitly in terms of the celebrated Catalan numbers, see Theorem 4.5. This remarkable combinatorial connection is key for establishing the algebraic structure of the spin $\mathrm{FH}$-algebra $\mathcal{K}$. Then the algebraic structure of $\mathcal{K}$ boils down to some novel combinatorial identity of Catalan numbers which is verified by using the Lagrange inversion formula. As a corollary, we obtain a new proof of a theorem in Brundan-Kleshchev [1] for the ring generators of $R S_{n}^{-}$, which has been used in modular spin representations of the symmetric groups.

Built on the results of Macdonald [8, pp.131-4], we develop a connection between the algebra $\mathcal{F}$ and the ring of symmetric functions. This is achieved by establishing an injective algebra homomorphism from $\mathcal{F}$ to $G$. 


\section{3}

It is well known that the 2-regular conjugacy classes of the symmetric group $S_{n}$ are parameterized by the odd partitions of $n$, and so at least formally there are many similarities between 2-modular representations and complex spin representations of the symmetric groups. John Murray studied the connections among Farahat-Higman, Jucys-Murphy, and center of the group algebra $F S_{n}$, where $F$ is a field of characteristic 2. In particular, a modulo 2 identity of Murray [10, Proposition 7.1] which involves the Catalan numbers bears an amazing resemblance to our Theorem 4.5 over integers. It would be very interesting to understand a conceptual connection behind these remarkable coincidences between 2-modular and complex spin setups for the symmetric groups.

\section{4}

The paper is organized as follows. In Section 2, we review the cycle notation for elements in the double cover $\widetilde{S}_{n}$ following [4] and the basis of even split class sums for the even center $\mathcal{Z}_{n}$. In Section 3, we establish the basic properties of the structure constants of $\mathcal{Z}_{n}$, which give rise to the filtered and graded spin FH-algebras. In Section 4, we develop the combinatorial connection between the odd Jucys-Murphy elements and Catalan numbers, and then use it in Section 5 to establish the main structure result for the filtered spin $\mathrm{FH}$-algebra $\mathcal{K}$. Finally in Section 6 , we develop a connection between the algebra $\mathcal{F}$ and symmetric functions.

\section{The preliminaries}

2.1 The double covers of the symmetric groups

The symmetric group $S_{n}$ is generated by $s_{i}, 1 \leq i \leq n-1$, subject to the relations

$$
s_{i}^{2}=1, \quad s_{i} s_{i+1} s_{i}=s_{i+1} s_{i} s_{i+1}, \quad s_{j} s_{i}=s_{i} s_{j} \text { for }|i-j|>1 .
$$

The generators $s_{i}$ may be identified with the transpositions $(i, i+1), 1 \leq i \leq n-1$. The double cover, $\widetilde{S}_{n}$, of $S_{n}$ is defined as the group generated by $t_{1}, t_{2}, \ldots, t_{n-1}$ and $z$, subject to the relations

$$
z \text { central, } t_{i}^{2}=z, \quad z^{2}=1, \quad t_{i} t_{i+1} t_{i}=t_{i+1} t_{i} t_{i+1}, \quad t_{j} t_{i}=z t_{i} t_{j} \text { for }|i-j|>1 .
$$

This gives rise to a short exact sequence of groups

$$
1 \longrightarrow\{1, z\} \longrightarrow \widetilde{S}_{n} \stackrel{\theta_{n}}{\longrightarrow} S_{n} \longrightarrow 1 \text {. }
$$

where $\theta_{n}\left(t_{i}\right)=s_{i}$.

There is a cycle presentation of $\widetilde{S}_{n}$, as there is for $S_{n}$. Following [4], define

$$
x_{i}=t_{i} t_{i+1} \cdots t_{n-1} t_{n} t_{n-1} \cdots t_{i+1} t_{i} \in \widetilde{S}_{n+1}
$$


for $i=1, \ldots, n-1$. Then, for a subset $\left\{i_{1}, \ldots, i_{m}\right\}$ of $\{1, \ldots, n\}$, we define a cycle of length $m$

$$
\left[i_{1}, i_{2}, \ldots, i_{m}\right]= \begin{cases}z, & \text { for } m=1, \\ x_{i_{1}} x_{i_{m}} x_{i_{m-1}} \cdots x_{i_{2}} x_{i_{1}}, & \text { for } m>1 .\end{cases}
$$

It follows that $\theta_{n}\left(\left[i_{1}, \ldots, i_{m}\right]\right)=\left(i_{1}, \ldots, i_{m}\right)$.

2.2 The even split conjugacy classes of $\widetilde{S}_{n}$

We sometimes write a partition $\lambda=\left(\lambda_{1}, \lambda_{2}, \ldots\right)$, a non-increasing sequence of positive integers, or write $\lambda=\left(1^{m_{1}}, 2^{m_{2}}, \ldots\right)=\left(i^{m_{i}}\right)_{i>1}$, where $m_{i}$ is the number of parts of $\lambda$ equal to $i$. We denote the length of $\lambda$ by $\ell(\lambda)$ and let $|\lambda|=\lambda_{1}+\lambda_{2}+\cdots$. Let $\mathcal{P}(n)$ (respectively, $\mathcal{E P}(n), \mathcal{O P}(n))$ denote the set of all partitions of $n$ (respectively, having only even, odd parts). Set $\mathcal{P}=\cup_{n \geq 0} \mathcal{P}(n), \mathcal{E P}=\cup_{n \geq 0} \mathcal{E P}(n)$, and $\mathcal{O P}=\cup_{n \geq 0} \mathcal{O P}(n)$.

Given $w \in S_{n}$ with cycle-type $\rho=\left(\rho_{1}, \ldots, \rho_{t}, 1, \ldots, 1\right)$, we define the modified cycle-type of $w$ to be $\tilde{\rho}=\left(\rho_{1}-1, \ldots, \rho_{t}-1\right)$ (see [8,pp.131]). Given a partition $\lambda$, let $C_{\lambda}(n)$ denote the conjugacy class of $S_{n}$ containing all elements of modified type $\lambda$ if $|\lambda|+\ell(\lambda) \leq n$, and denote $C_{\lambda}(n)=\emptyset$ otherwise.

What follows is, essentially, a "modified type" version of what appears in [4, Section 3B]. Also we have adopted the more standard modern convention of group multiplication from right to left (different from the convention in [4, 12]), e.g. $(1,2)(2,3)=(1,2,3)$ (instead of $(1,3,2))$. The set $\theta_{n}^{-1}\left(C_{\lambda}(n)\right)$ either splits into two conjugacy classes of $\widetilde{S}_{n}$, or it is a single conjugacy class of $\widetilde{S}_{n}$. In the first case, we call $C_{\lambda}(n)$ a split conjugacy class of $S_{n}$, and call the two conjugacy classes in $\theta_{n}^{-1}\left(C_{\lambda}(n)\right)$ split conjugacy classes of $\widetilde{S}_{n}$. In fact, we have the following (cf. [4, Theorem 3.6]).

Lemma 2.1 Let $C_{\lambda}(n)$ be a nonempty conjugacy class of $S_{n}$. Then $\theta_{n}^{-1}\left(C_{\lambda}(n)\right)$ splits into two conjugacy classes of $\widetilde{S}_{n}$ if and only if either

(1) $\lambda \in \mathcal{E P}$ (whence the conjugacy class or its elements are called even split), or

(2) $|\lambda|$ is odd, all parts of $\lambda$ are distinct, and $|\lambda|+\ell(\lambda)=n$ or $n-1$.

For $\lambda \in \mathcal{E P}$, denote by $D_{\lambda}(n)$ the (even split) conjugacy class in $\widetilde{S}_{n}$ which contains the following distinguished element of modified type $\lambda$

$$
\begin{aligned}
t_{\lambda}= & {\left[1,2, \ldots, \lambda_{1}+1\right]\left[\lambda_{1}+2, \ldots, \lambda_{1}+\lambda_{2}+2\right] } \\
& \ldots\left[\lambda_{1}+\ldots+\lambda_{\ell-1}+\ell, \ldots, \lambda_{1}+\ldots+\lambda_{\ell}+\ell\right]
\end{aligned}
$$

and hence $\theta_{n}^{-1}\left(C_{\lambda}(n)\right)=D_{\lambda}(n) \bigsqcup z D_{\lambda}(n)$ if $|\lambda|+\ell(\lambda) \leq n$; set $D_{\lambda}(n)=\emptyset$ otherwise.

Suppose $s^{\prime} \in \theta_{n}^{-1}(s)$. Then, the other member of $\theta_{n}^{-1}(s)$ is $z s^{\prime}$. We denote the common value of $s^{\prime} x s^{-1}=\left(z s^{\prime}\right) x\left(z s^{\prime}\right)^{-1}$ by $s x s^{-1}$, for $x \in \widetilde{S}_{n}$. Let $x=$ $\left[i_{1}, \ldots, i_{m}\right]\left[j_{1}, \ldots, j_{k}\right] \cdots \in \widetilde{S}_{n}$ be a product of disjoint cycles such that $\theta_{n}(x)$ is of modified type $\lambda$. Let $s \in S_{n}$ be of modified type $\mu$. Then

$$
s\left(\left[i_{1}, \ldots, i_{m}\right]\left[j_{1}, \ldots, j_{k}\right] \cdots\right) s^{-1}=z^{|\lambda||\mu|}\left[s\left(i_{1}\right), \ldots, s\left(i_{m}\right)\right]\left[s\left(j_{1}\right), \ldots, s\left(j_{k}\right)\right] \cdots,
$$


according to [4, Proposition 3.5]. In particular, we have the following.

Lemma 2.2 For $\lambda \in \mathcal{E P}$ of length $\ell$, the even split conjugacy class $D_{\lambda}(n)$ consists of all products of disjoint cycles in $\widetilde{S}_{n}$ of the form

$$
\left[i_{1}, \ldots, i_{\lambda_{1}+1}\right]\left[j_{1}, \ldots, j_{\lambda_{2}+1}\right] \cdots\left[k_{1}, \ldots, k_{\lambda_{\ell}+1}\right] .
$$

Remark 2.3 The $D_{\lambda}(n)$ in the present paper is different from that in [4]. The corresponding conjugacy class in [4] - call it $\tilde{D}_{\lambda}(n)$ to distinguish from our $D_{\lambda}(n)$ - is given by $\tilde{D}_{\lambda}(n)=z^{n-\ell(\lambda)} D_{\lambda}(n)$. The present definition of $D_{\lambda}(n)$, just as the definition of modified cycle type, is consistent with the natural embedding of $\widetilde{S}_{n}$ in $\widetilde{S}_{n+1}$ in the sense that $D_{\lambda}(n+1) \cap \widetilde{S}_{n}=D_{\lambda}(n)$ for all $n$.

The embedding of $\widetilde{S}_{n}$ in $\widetilde{S}_{n+1}$ gives the union $\widetilde{S}_{\infty}=\cup_{n \geq 1} \widetilde{S}_{n}$ a natural group structure. By Remark 2.3, the union $D_{\lambda}:=\cup_{n \geq 1} D_{\lambda}(n)$ is an even split conjugacy class in $\widetilde{S}_{\infty}$ for each $\lambda \in \mathcal{E} \mathcal{P}$. We define the homomorphism $\theta: \widetilde{S}_{\infty} \longrightarrow S_{\infty}$ by $\theta(x)=\theta_{n}(x)$ for $x$ in $\widetilde{S}_{n}$.

\subsection{The spin group superalgebra}

Let $R$ be a commutative ring which contains no 2-torsion. The group algebra $R \widetilde{S}_{n}$ has a super (i.e. $\mathbb{Z}_{2}$-graded) algebra structure given by declaring the elements $t_{i}$ for all $1 \leq i \leq n-1$ to be odd, i.e., of degree 1. The spin group algebra, $R S_{n}^{-}$is defined to be the quotient of $R \widetilde{S}_{n}$ by the ideal generated by $z+1$ :

$$
R S_{n}^{-}=R \widetilde{S}_{n} /\langle z+1\rangle
$$

We may view the spin group algebra as the algebra generated by the elements $t_{i}$, $i=1, \ldots, n-1$, subject to the relations

$$
t_{i}^{2}=-1, \quad t_{i} t_{i+1} t_{i}=t_{i+1} t_{i} t_{i+1}, \quad t_{j} t_{i}=-t_{i} t_{j} \quad(|i-j|>1) .
$$

Remark 2.4 One defining relation of $R S_{n}^{-}$above is $t_{i}^{2}=-1$ as in [4], while a different relation $t_{i}^{2}=1$ was used in the recent book of Kleshchev [7] and in [16]. One can use a scalar $\sqrt{-1}$ to exchange the two notations. Also, the cycle notation in $R S_{n}^{-}$adopted in [4] and here differs from the one used in $[7,13]$ by some scalars dependent on the length of a cycle.

We adapt the notion of cycles for $\widetilde{S}_{n}$ to the spin group algebra setting. As before, and by an abuse of notation, we take

$$
x_{i}=t_{i} t_{i+1} \cdots t_{n-2} t_{n-1} t_{n-2} \cdots t_{i+1} t_{i}
$$

for $i=1, \cdots, n-1$. Then for a subset $\left\{i_{1}, \ldots, i_{m}\right\}$ of $\{1, \ldots, n\}$, a cycle in the spin group algebra is defined to be

$$
\left[i_{1}, i_{2}, \ldots, i_{m}\right]= \begin{cases}-1, & \text { for } m=1, \\ x_{i_{1}} x_{i_{m}} x_{i_{m-1}} \cdots x_{i_{2}} x_{i_{1}}, & \text { for } m>1 .\end{cases}
$$


We have the following properties of the cycles (cf. [4, Theorem 3.1]):

$$
\begin{aligned}
{\left[i_{1}, \ldots, i_{m}\right]=} & (-1)^{m-1}\left[i_{2}, \ldots, i_{m}, i_{1}\right], \\
{[i, i+1, \ldots, i+j-1]=} & (-1)^{j-1} t_{i} t_{i+1} \ldots t_{i+j-2}, \quad j>0, \\
{\left[a, i_{1}, \ldots, i_{m}\right]\left[a, j_{1}, \ldots, j_{n}\right]=} & -\left[a, j_{1}, \ldots, j_{n}, i_{1}, \ldots, i_{m}\right], \\
& \text { if }\left\{i_{1}, \ldots, i_{m}\right\} \cap\left\{j_{1}, \ldots, j_{n}\right\}=\emptyset .
\end{aligned}
$$

The algebra $R S_{n}^{-}$inherits a superalgebra structure from $R \widetilde{S}_{n}$ by letting the elements $t_{i}, 1 \leq i \leq n-1$ be of degree 1 . Denote by $\mathcal{Z}_{n}=\mathcal{Z}\left(R S_{n}^{-}\right)$the even center of $R S_{n}^{-}$, i.e. the set of even central elements in the superalgebra $R S_{n}^{-}$, for finite $n$ as well as $n=\infty$. For $\lambda \in \mathcal{E} \mathcal{P}$, we let $d_{\lambda}(n)$ denote the image in $R S_{n}^{-}$of the class sum of $D_{\lambda}(n)$ in $R \widetilde{S}_{n}$ if $|\lambda|+\ell(\lambda) \leq n$, and 0 otherwise. The following is clear.

Lemma 2.5 The set $\left\{d_{\lambda}(n)|\lambda \in \mathcal{E P},| \lambda \mid+\ell(\lambda) \leq n\right\}$ forms a basis for the even center $\mathcal{Z}_{n}$.

\section{The structure constants of the centers}

\subsection{Some preparatory lemmas}

Let $\mathbb{N}$ be the set of positive integers. For any subset $Y$ of elements in $\widetilde{S}_{\infty}$, define a subset of $\mathbb{N}$

$$
\mathbb{N}(Y)=\{j \in \mathbb{N} \mid \theta(\sigma)(j) \neq j \text { for some } \sigma \text { in } Y\} .
$$

It is clear that $\mathbb{N}(Y)=\cup_{\sigma \in Y} \mathbb{N}(\sigma)$. We denote the cardinality of $\mathbb{N}(Y)$ by $|\mathbb{N}(Y)|$.

Lemma 3.1 [2, Lemma 3.5] Let $x, y \in \widetilde{S}_{\infty}$. Suppose that $x$ is of modified type $\lambda, y$ is of modified type $\mu$ and that $x y$ is of modified type $v$. Then $|\nu| \leq|\lambda|+|\mu|$, with equality if and only if $\mathbb{N}(x y)=\mathbb{N}(x, y)$.

Two $r$-tuples of elements in $\widetilde{S}_{\infty},\left(x_{1}, \ldots, x_{r}\right)$ and $\left(y_{1}, \ldots, y_{r}\right)$, are said to be conjugate in $\widetilde{S}_{\infty}$ if $y_{i}=w x_{i} w^{-1}, 1 \leq i \leq r$ for some $w$ in $\widetilde{S}_{\infty}$. For any conjugacy class, $\mathcal{C}$, of such $r$-tuples, we denote by $|\mathbb{N}(\mathcal{C})|$ the cardinality of $\mathbb{N}\left(x_{1}, \ldots, x_{n}\right)$ for any element $\left(x_{1}, \ldots, x_{n}\right)$ in $\mathcal{C}$.

The next lemma is a spin variant of [2, Lemma 2.1].

Lemma 3.2 Let $\mathcal{C}$ be a conjugacy class of $r$-tuples of even split elements in $\widetilde{S}_{\infty}$. Then, the intersection of $\mathcal{C}$ with $\overbrace{\widetilde{S}_{n} \times \cdots \times \widetilde{S}_{n}}^{r}$ is empty if $n<|\mathbb{N}(\mathcal{C})|$ and is a conjugacy class of $r$-tuples in $\widetilde{S}_{n}$ if $n \geq|\mathbb{N}(\mathcal{C})|$. The number of $r$-tuples in the intersection is $n(n-1) \cdots(n-|\mathbb{N}(\mathcal{C})|+1) / k(\mathcal{C})$ where $k(\mathcal{C})$ is a constant. 


\subsection{Behavior of the structure constants}

Consider the multiplication in the even center $\mathcal{Z}_{n}$ :

$$
d_{\lambda}(n) d_{\mu}(n)=\sum_{\nu \in \mathcal{E} \mathcal{P}} a_{\lambda \mu}^{v}(n) d_{\nu}(n)
$$

where the structure constants $a_{\lambda \mu}^{\nu}(n)$ are integers and they are undetermined when $n<|v|+\ell(v)$.

Example 3.3 Denote by $c_{\lambda}(n)$ the class sum of $C_{\lambda}(n)$ for $S_{n}$. Then,

$$
\begin{aligned}
c_{(4)}(8) c_{(2)}(8)=25 c_{(4)}(8) & +35 c_{(2)}(8)+32 c_{(3,1)}(8)+32 c_{(1,1)}(8)+18 c_{(2,2)}(8) \\
& +7 c_{(6)}(8)+2 c_{(4,2)}(8) \in \mathcal{Z}\left(R S_{8}\right), \\
d_{(4)}(8) d_{(2)}(8)=13 d_{(4)}(8) & -35 d_{(2)}(8)-18 d_{(2,2)}(8) \\
& -7 d_{(6)}(8)+2 d_{(4,2)}(8) \in \mathcal{Z}\left(R S_{8}^{-}\right), \\
{\left[c_{(2)}(6)\right]^{2}=2 c_{(2,2)}(6) } & +5 c_{(4)}(6)+10 c_{(2)}(6)+8 c_{(1,1)}(6)+40 c_{\emptyset}(6) \in \mathcal{Z}\left(R S_{6}\right), \\
{\left[d_{(2)}(6)\right]^{2}=} & 2 d_{(2,2)}(6)-5 d_{(4)}(6)+8 d_{(2)}(6)+40 d_{\emptyset}(6) \in \mathcal{Z}\left(R S_{6}^{-}\right) .
\end{aligned}
$$

The following is a spin version of [2, Theorem 2.2 and Lemma 3.9], and we present its detailed proof to indicate the sign difference from loc. cit.

Theorem 3.4 Let $\lambda, \mu, v \in \mathcal{E} \mathcal{P}$.

(1) There is a unique polynomial $f_{\lambda \mu}^{\nu}(x)$ such that $a_{\lambda \mu}^{\nu}(n)=f_{\lambda \mu}^{\nu}(n)$ for all $n \geq|\nu|+$ $\ell(\nu)$. The degree of $f_{\lambda \mu}^{\nu}(x)$ is no greater than the maximum value of $|\mathbb{N}(\mathcal{C})|-$ $|\nu|-\ell(\nu)$ for any class $\mathcal{C}$ of pairs $(a, b)$ such that $a \in D_{\lambda}, b \in D_{\mu}$, and either $a b \in D_{v}$ or $a b \in z D_{v}$.

(2) The polynomial $f_{\lambda \mu}^{\nu}(x)=0$, unless $|\nu| \leq|\lambda|+|\mu|$.

(3) If $|\nu|=|\lambda|+|\mu|$, then the polynomial $f_{\lambda \mu}^{\nu}(x)$ is constant, i.e., the structure constants $a_{\lambda \mu}^{v}(n)$ are independent of $n$.

Proof Let $\tilde{d}_{\lambda}(n)$ be the class sum of $D_{\lambda}(n)$ in $R \widetilde{S}_{n}$. We write

$$
\tilde{d}_{\lambda}(n) \tilde{d}_{\mu}(n)=\sum_{\nu} u_{\lambda \mu}^{\nu}(n) \tilde{d}_{\nu}(n)+\sum_{\nu} v_{\lambda \mu}^{\nu}(n) z \tilde{d}_{\nu}(n) .
$$

Therefore, upon passing to $R S_{n}^{-}$, we obtain that

$$
a_{\lambda \mu}^{v}(n)=u_{\lambda \mu}^{v}(n)-v_{\lambda \mu}^{v}(n)
$$

For each triple $(\lambda, \mu, \nu)$ of partitions in $\mathcal{E} \mathcal{P}$, consider the sets

$$
\begin{aligned}
X_{\lambda \mu}^{\nu} & =\left\{(a, b) \in \widetilde{S}_{\infty} \times \widetilde{S}_{\infty} \mid a \in D_{\lambda}, b \in D_{\mu} \text { and } a b \in D_{\nu}\right\}, \\
Y_{\lambda \mu}^{\nu} & =\left\{(a, b) \in \widetilde{S}_{\infty} \times \widetilde{S}_{\infty} \mid a \in D_{\lambda}, b \in D_{\mu} \text { and } a b \in z D_{\nu}\right\} .
\end{aligned}
$$


Any conjugate pair of $(a, b) \in X_{\lambda \mu}^{\nu}$ (resp. $\left.Y_{\lambda \mu}^{\nu}\right)$ also lies in $X_{\lambda \mu}^{\nu}$ (resp. $Y_{\lambda \mu}^{\nu}$ ). Therefore, $X_{\lambda \mu}^{v}$ and $Y_{\lambda \mu}^{v}$ can be written as disjoint unions of conjugacy classes, say

$$
\begin{aligned}
X_{\lambda \mu}^{v} & =\mathcal{C}_{1} \sqcup \mathcal{C}_{2} \sqcup \cdots \sqcup \mathcal{C}_{r}, \\
Y_{\lambda \mu}^{v} & =\mathcal{C}_{r+1} \sqcup \mathcal{C}_{r+2} \sqcup \cdots \sqcup \mathcal{C}_{r+s} .
\end{aligned}
$$

By Lemma 3.2, the number of pairs $(a, b)$ of $X_{\lambda \mu}^{\nu}$ with $a$ and $b$ in $\widetilde{S}_{n}$ is

$$
\sum_{i=1}^{r}\left(n(n-1) \cdots\left(n-\left|\mathbb{N}\left(\mathcal{C}_{i}\right)\right|+1\right)\right) / k\left(\mathcal{C}_{i}\right) .
$$

In other words, the above number is equal to the total number of elements from $D_{v}(n)$ that appear upon multiplication of the class sums $\tilde{d}_{\lambda}(n)$ and $\tilde{d}_{\mu}(n)$. To find $u_{\lambda \mu}^{v}(n)$, we must divide (3.1) by the order of $D_{v}(n)$, which is equal to

$$
(n(n-1) \cdots(n-|v|-\ell(v)+1)) / k(v) .
$$

Note that $|\nu|+\ell(v)=|\mathbb{N}(a b)| \leq|\mathbb{N}(a, b)|=\left|\mathbb{N}\left(\mathcal{C}_{i}\right)\right|$ for $(a, b) \in \mathcal{C}_{i}$. We have $u_{\lambda \mu}^{v}(n)=k(v) \sum_{i=1}^{r}\left((n-|v|-\ell(v))(n-|v|-\ell(v)-1) \cdots\left(n-\left|\mathbb{N}\left(\mathcal{C}_{i}\right)\right|+1\right)\right) / k\left(\mathcal{C}_{i}\right)$.

One has a similar formula for $v_{\lambda \mu}^{v}(n)$. So the required polynomial $f_{\lambda \mu}^{\nu}(x)$ is given by

$$
\begin{array}{r}
\sum_{i=1}^{r} \frac{k(v)}{k\left(\mathcal{C}_{i}\right)}\left((x-|v|-\ell(v))(x-|v|-\ell(v)-1) \cdots\left(x-\left|\mathbb{N}\left(\mathcal{C}_{i}\right)\right|+1\right)\right) \\
-\sum_{i=r+1}^{r+s} \frac{k(v)}{k\left(\mathcal{C}_{i}\right)}\left((x-|v|-\ell(v))(x-|v|-\ell(v)-1) \cdots\left(x-\left|\mathbb{N}\left(\mathcal{C}_{i}\right)\right|+1\right)\right)
\end{array}
$$

whose degree is no greater than $\max _{1 \leq i \leq r+s}\left\{\left|\mathbb{N}\left(\mathcal{C}_{i}\right)\right|-|\nu|-\ell(\nu)\right\}$. This proves (1).

Part (2) holds, since $a_{\lambda \mu}^{\nu}(n)=0$ for every $n$ unless $|\nu| \leq|\lambda|+|\mu|$ by Lemma 3.1. If $|\nu|=|\lambda|+|\mu|$, it follows by (1) and Lemma 3.1 that the polynomial $f_{\lambda \mu}^{\nu}$ is of degree 0 , whence a constant. This proves (3).

\subsection{The spin FH-algebras $\mathcal{K}$ and $\mathcal{F}$}

Let $\mathbb{B}$ be the ring consisting of all polynomials that take integer values at all integers. By definition, $f_{\lambda \mu}^{\nu}(x)$ belongs to $\mathbb{B}$ for all $\lambda, \mu, v$ in $\mathcal{E P}$. We define a $\mathbb{B}$-algebra $\mathcal{K}$ with $\mathbb{B}$-basis $\left\{d_{\lambda} \mid \lambda \in \mathcal{E} \mathcal{P}\right\}$ and multiplication given by

$$
d_{\lambda} d_{\mu}=\sum_{\nu} f_{\lambda \mu}^{v}(x) d_{\nu}
$$

where the sum is over all $\nu$ in $\mathcal{E} \mathcal{P}$ such that $|\nu| \leq|\lambda|+|\mu|$. We will refer to $\mathcal{K}$ as the (filtered) spin FH-algebra. The following is an analogue of [2, Theorem 2.4] and it can be proved in the same elementary way. 
Proposition 3.5 The spin $\mathrm{FH}$-algebra $\mathcal{K}$ is associative and commutative. There exists a surjective homomorphism of algebras

$$
\phi_{n}: \mathcal{K} \longrightarrow \mathcal{Z}\left(\mathbb{Z} S_{n}^{-}\right), \quad \sum f_{\lambda}(x) d_{\lambda} \mapsto \sum f_{\lambda}(n) d_{\lambda}(n) .
$$

Let $\mathcal{K}^{(m)}$ with $m$ being even be the subspace of $\mathcal{K}$ that is the $\mathbb{B}$-span of all $d_{\lambda}$ 's with $|\lambda|=m$ (in this case, $m$ will be called the degree of $d_{\lambda}$ ). Set $\mathcal{K}_{(m)}=$ $\oplus_{0 \leq i \leq m, i \text { even }} \mathcal{K}^{(i)}$. Then, it follows by Theorem 3.4 that $\left\{\mathcal{K}_{(m)}\right\}$ defines a filtered algebra structure on $\mathcal{K}$. Given $x \in \mathcal{K}$, there is a unique even integer $m$ such that $x \in \mathcal{K}_{(m)}$ and $x \notin \mathcal{K}_{(m-2)}$, and we denote by $x^{*}$ the top degree part of $x$ such that $x-x^{*} \in \mathcal{K}_{(m-2)}$. Hence, we have

$$
\left(d_{\lambda} d_{\mu}\right)^{*}=\sum_{|\nu|=|\lambda|+|\mu|} f_{\lambda \mu}^{\nu} d_{\nu} \in \mathcal{K} .
$$

Define a graded $\mathbb{Z}$-algebra, $\mathcal{F} \simeq \mathcal{F}^{(0)} \oplus \mathcal{F}^{(2)} \oplus \mathcal{F}^{(4)} \oplus \cdots$, where $\mathcal{F}^{(m)}$ ( $m$ even) is defined to be the $\mathbb{Z}$-span of all the symbols $d_{\lambda}$ with $|\lambda|=m$, with multiplication

$$
d_{\lambda} * d_{\mu}=\sum_{|v|=|\lambda|+|\mu|} f_{\lambda \mu}^{\nu} d_{\nu}
$$

Recall that the coefficients $f_{\lambda \mu}^{\nu}$ such that $|\nu|=|\lambda|+|\mu|$ (which are equal to $a_{\lambda \mu}^{\nu}(n)$ for large $n$ ) are integers. Then the graded algebra associated to $\mathcal{K}$ is given by

$$
\operatorname{gr} \mathcal{K} \cong \mathbb{B} \otimes_{\mathbb{Z}} \mathcal{F}
$$

It follows from Proposition 3.5 that the algebra $\mathcal{F}$ is commutative and associative. We will refer to $\mathcal{F}$ as the graded spin FH-algebra.

It will be convenient to think of $d_{\lambda}$ in $\mathcal{K}$ or $\mathcal{F}$ as the class sum of the conjugacy class $D_{\lambda}$ in $\mathcal{Z}_{\infty}$, but with multiplications modified in two different ways; note that the usual multiplication on $\mathcal{Z}_{\infty}$ induced from the group multiplication in $\widetilde{S}_{\infty}$ has an obvious divergence problem.

Lemma 3.1 also exhibits filtered ring structures on $R S_{n}^{-}$as well as on $\mathcal{Z}_{n}$, which allow us to define the associated graded rings $\operatorname{gr}\left(R S_{n}^{-}\right)$and $\operatorname{gr} \mathcal{Z}_{n}$. Thus, it will make sense to talk about the top degree term $x^{*}$ of $x \in R S_{n}^{-}$.

\subsection{Some distinguished structure constants}

As in [8], we will write $\lambda \cup \mu$ for the partition that is the union of $\lambda$ and $\mu$. If $\mu$ is a partition contained in $\lambda$, the notation $\lambda-\mu$ will denote the partition obtained by deleting all parts of $\mu$ from $\lambda$. Let $\unrhd$ denote the usual dominance order of partitions. For a one-part partition $(s)$ with even $s$, we write $d_{(s)}=d_{s}$.

Proposition 3.6 Let $\lambda=\left(i^{m_{i}(\lambda)}\right)_{i \geq 1} \in \mathcal{E} \mathcal{P}$ and let $s>0$ be even. Then in $\mathcal{K}$, we have

$$
\left(d_{\lambda} d_{s}\right)^{*}=\sum_{\mu}(-1)^{\ell(\mu)} \frac{\left(m_{s+|\mu|}+1\right)(s+|\mu|+1) s !}{m_{0}(\mu) ! \prod_{i \geq 1} m_{i}(\mu) !} d_{\lambda \cup(s+|\mu|)-\mu}
$$


where the sum is over all partitions $\mu=\left(i^{m_{i}(\mu)}\right)_{i \geq 1} \in \mathcal{E P}$ contained in $\lambda$ with $\ell(\mu) \leq$ $(s+1)$, and $m_{0}(\mu)=s+1-\ell(\mu)$.

Proof The proof is completely analogous to the proof of [2, Lemma 3.11], and hence will be omitted. Here we only remark that the sign $(-1)^{\ell(\mu)}$ appearing on the righthand side of (3.2) is due to the multiplication of $\ell(\mu)$ cycles in $d_{\lambda}$ with an $(s+1)$ cycle and (2.2).

In the same way as Macdonald ([8, pp.132]) reformulated the formula in [2], Proposition 3.6 can be reformulated as follows.

Proposition 3.7 Let $\lambda=\left(i^{m_{i}(\lambda)}\right)_{i \geq 1} \in \mathcal{E} \mathcal{P}$ and let $s>0$ be even.

(1) If $|\lambda|+s=m$, then

$$
f_{\lambda(s)}^{(m)}= \begin{cases}\frac{(-1)^{\ell(\lambda)}(m+1) s !}{(s+1-\ell(\lambda)) ! \prod_{i \geq 1} m_{i}(\lambda) !}, & \text { if } \ell(\lambda) \leq s+1, \\ 0, & \text { otherwise. }\end{cases}
$$

(2) If $|\lambda|+s=|v|$, and write $v=\left(v_{1}, v_{2}, \ldots\right)$, then

$$
f_{\lambda(s)}^{\nu}=\sum f_{\mu(s)}^{\left(v_{i}\right)}
$$

summed over pairs $(i, \mu)$ such that $\mu \cup v=\lambda \cup\left(v_{i}\right)$, where $\mu \in \mathcal{E} \mathcal{P}$.

(3) The coefficient $f_{\lambda(s)}^{\nu}=0$ unless $\nu \unrhd \lambda \cup(s)$, and $f_{\lambda(s)}^{\lambda \cup(s)}>0$.

Remark 3.8 Let $\lambda=\left(i^{m_{i}(\lambda)}\right)_{i \geq 1}$ and $\mu=\left(i^{m_{i}(\mu)}\right)_{i \geq 1}$ be partitions in $\mathcal{E} \mathcal{P}$. As a variant of [2, Lemma 3.10], we have the following formula of structure constants:

$$
f_{\lambda \mu}^{\lambda \cup \mu}=\prod_{i \geq 1} \frac{\left(m_{i}(\lambda)+m_{i}(\mu)\right) !}{m_{i}(\lambda) ! m_{i}(\mu) !}
$$

Theorem 3.9 The algebra $\mathbb{Q} \otimes_{\mathbb{Z}} \mathcal{F}$ is a polynomial algebra generated by $d_{m}$ with $m=2,4,6, \ldots$.

Proof Let $\lambda=\left(\lambda_{1}, \lambda_{2}, \ldots\right) \in \mathcal{E} \mathcal{P}$. By Proposition 3.7, we have inductively

$$
d_{\lambda_{1}} * d_{\lambda_{2}} * \cdots=\sum_{\mu \in \mathcal{E} \mathcal{P}, \mu \unrhd \lambda} h_{\lambda \mu} d_{\mu}
$$

where $h_{\lambda \mu} \in \mathbb{Z}$ with $h_{\lambda \lambda}>0$ by Remark 3.8. Hence $\left(h_{\lambda \mu}\right)$ is a triangular integral matrix with nonzero diagonal entries, whose inverse matrix has rational entries. 


\section{The Jucys-Murphy elements and Catalan numbers}

\subsection{The odd Jucys-Murphy elements}

Recall the Jucys-Murphy elements [6,9] in the symmetric group algebra $R S_{n}$ are defined to be $\xi_{k}=\sum_{i=1}^{k-1}(i, k)$ for $1 \leq k \leq n$. The odd Jucys-Murphy elements $M_{k}$ in the spin group algebra $R S_{n}^{-}$were introduced by Sergeev [13] up to a common factor $\sqrt{-1}$ (cf. Remark 2.4) as

$$
M_{k}=\sum_{i=1}^{k-1}[i, k], \quad 1 \leq k \leq n
$$

and they are closely related to the constructions in $[11,16]$. It follows by $(2.2)$ that

$$
M_{k}^{2}=-(k-1)-\sum_{i, j=1 ; i \neq j}^{k-1}[i, j, k] .
$$

We caution that our $M_{k}^{2}$ differs from the square of the odd Jucys-Murphy elements used in $[1,7,13,16]$ by a sign.

According to $[11,13]$, for $1 \leq r \leq n$, the $r$ th elementary symmetric function in the $M_{k}^{2}(1 \leq k \leq n)$

$$
e_{r ; n}=\sum_{1 \leq i_{1}<i_{2}<\cdots<i_{r} \leq n} M_{i_{1}}^{2} M_{i_{2}}^{2} \cdots M_{i_{r}}^{2}
$$

lies in the even center $\mathcal{Z}_{n}$. For $\lambda \in \mathcal{E P}(2 r)$, we note that the coefficient in $e_{r ; n}$ of the given element $t_{\lambda}$ introduced in (2.1) (for $n \geq|\lambda|+\ell(\lambda)$ ) is independent of $n$. Hence, we have the following well-defined element

$$
e_{r}^{*}=\sum_{i_{1}<i_{2}<\cdots<i_{r}}\left(M_{i_{1}}^{2} M_{i_{2}}^{2} \cdots M_{i_{r}}^{2}\right)^{*}
$$

as the $n \rightarrow \infty$ limit of $e_{r ; n}^{*}$, which is then written as

$$
e_{r}^{*}=\sum_{\lambda \in \mathcal{E} \mathcal{P}(2 r)} A_{\lambda} d_{\lambda} \in \mathcal{K} .
$$

We shall write $A_{2 r}$ for $A_{(2 r)}$. The goal of this section is to calculate the $A_{\lambda}$.

Example 4.1 The first few $e_{r}^{*}$ are computed as follows.

$$
\begin{aligned}
& e_{1}^{*}=-d_{2} \\
& e_{2}^{*}=d_{(2,2)}-2 d_{4} \\
& e_{3}^{*}=-d_{(2,2,2)}+2 d_{(4,2)}-5 d_{6} .
\end{aligned}
$$


4.2 The relations among $A_{\lambda}$

Lemma 4.2 Let $r \geq 1$ and $\lambda=\left(\lambda_{1}, \lambda_{2}, \ldots, \lambda_{\ell}\right) \in \mathcal{E P}(2 r)$ be of length $\ell$. The coefficients $A_{\lambda}$ in (4.2) admit the following factorization property:

$$
A_{\lambda}=A_{\lambda_{1}} A_{\lambda_{2}} \cdots A_{\lambda_{\ell}} .
$$

Proof The proof is by induction on $\ell(\lambda)=\ell$, with the case $\ell(\lambda)=1$ being trivial.

Let $\lambda \in \mathcal{E} \mathcal{P}(2 r)$ be of length $\ell>1$. Recall that the element $t_{\lambda}$ in (2.1) of modified type $\lambda=\left(\lambda_{1}, \lambda_{2}, \ldots, \lambda_{\ell}\right)$ can be written as $t_{\lambda}=u v$, where

$$
u=\left[1,2, \ldots, \lambda_{1}+1\right] \cdots\left[\lambda_{1}+\cdots+\lambda_{\ell-2}+\ell-1, \ldots, \lambda_{1}+\cdots+\lambda_{\ell-1}+\ell-1\right]
$$

is of modified type $\tilde{\lambda}=\left(\lambda_{1}, \lambda_{2}, \ldots, \lambda_{\ell-1}\right)$ and length $(\ell-1)$, and

$$
v=\left[\lambda_{1}+\cdots+\lambda_{\ell-1}+\ell, \ldots, \lambda_{1}+\cdots+\lambda_{\ell}+\ell\right] .
$$

To find the coefficient $A_{\lambda}$ of $d_{\lambda}$ in $e_{r}^{*}$ we will count the number of appearances of $t_{\lambda}$ in $e_{r}^{*}$. Suppose that $t_{\lambda}$ appears upon multiplication of a particular term, $M_{i_{1}}^{2} \cdots M_{i_{r}}^{2}$, of $e_{r}^{*}$. Since $t_{\lambda}$ has the top degree $2 r$ among the cycles in $M_{i_{1}}^{2} \cdots M_{i_{r}}^{2}$, we can and will regard the products $M_{i_{s}}^{2}$ and $M_{i_{1}}^{2} \cdots M_{i_{r}}^{2}$ as in $\operatorname{gr}\left(R S_{n}^{-}\right)$in the remainder of this proof, e.g. $M_{k}^{2}=-\sum_{1 \leq i \neq j \leq k-1}[i, j, k]$. To produce a $(2 n+1)$-cycle by multiplying together 3-cycles, one needs $n 3$-cycles. Then we have that $i_{r}=\lambda_{1}+\cdots+\lambda_{\ell}+\ell$. Furthermore, since $i_{1}<\cdots<i_{r}$ and because of the increasing arrangement of the cycles of $u$ and $v$, the cycle $v$ must appear upon multiplication of the last $\lambda_{\ell} / 2$ of the $M_{i_{k}}^{2}$, and the smallest such $i_{k}$ has to satisfy $i_{k} \geq \lambda_{1}+\cdots+\lambda_{\ell-1}+\ell+2$. The factor $u$ must appear upon multiplication of the first $\left(\lambda_{1}+\cdots+\lambda_{\ell-1}\right) / 2$ factors $M_{i_{k}}^{2}$ with the largest such $i_{k}$ being $\lambda_{1}+\cdots+\lambda_{\ell-1}+\ell-1$.

Therefore, the contribution to the multiplicity of $t_{\lambda}$ in $e_{r}$ comes exactly from the partial sum $\sum M_{i_{1}}^{2} \cdots M_{i_{r}}^{2}$ of $e_{r}$ where the indices satisfy the conditions

$$
\begin{aligned}
3 & \leq i_{1}<i_{2}<\cdots<\left(i_{r-\frac{1}{2} \lambda_{\ell}}=\lambda_{1}+\cdots+\lambda_{\ell-1}+\ell-1\right) \\
& <i_{r-\frac{1}{2} \lambda_{\ell}+1}<\cdots<i_{r}=\lambda_{1}+\cdots+\lambda_{\ell}+\ell
\end{aligned}
$$

and $i_{r-\lambda_{\ell} / 2+1} \geq \lambda_{1}+\cdots+\lambda_{\ell-1}+\ell+2$. This partial sum can be written as the product $E_{1} E_{2}$, where

$$
E_{1}=\sum M_{i_{1}}^{2} \cdots M_{i_{r-\frac{1}{2} \lambda_{\ell}}^{2}}^{2}, \quad E_{2}=\sum M_{i_{r-\frac{1}{2} \lambda_{\ell}+1}^{2}}^{2} \cdots M_{i_{r}}^{2} .
$$

Thus, the multiplicity of $t_{\lambda}$ in $e_{r}^{*}$ is the product of the coefficient of $u$ in $E_{1}$, which is $\prod_{i=1}^{\ell-1} A_{\lambda_{i}}$ by the induction assumption, and the coefficient of $v$ in $E_{2}$, which is $A_{\lambda_{\ell}}$. This proves the lemma.

Lemma 4.3 The following recursive relation holds:

$$
A_{2}=-1, \quad A_{2 r}=2 A_{2 r-2}-\sum_{s=1}^{r-2} A_{(2 r-2-2 s, 2 s)} \quad(r \geq 2)
$$


where we have denoted $A_{(b, a)}=A_{(a, b)}$ for $a>b$.

Proof We have seen that $A_{2}=-1$ in Example 4.1. Note that $A_{2 r}$ is equal to the coefficient of $d_{(2 r)}(2 r+1)$ appearing in $e_{r}^{*}$, which is the multiplicity of each $(2 r+1)$ cycle, say $[1,2, \cdots, 2 r+1]$, that appears in $e_{r ; 2 r+1}$. Observe that only the following summand

$$
\sum_{1 \leq i_{1}<\cdots<i_{r-1} \leq 2 r} M_{i_{1}}^{2} \cdots M_{i_{r-1}}^{2} \cdot M_{2 r+1}^{2}
$$

in $e_{r ; 2 r+1}$ contributes to the multiplicity of the $(2 r+1)$-cycle $[1,2, \cdots, 2 r+1]$. The expression $\sum_{i_{1}<\cdots<i_{r-1} \leq 2 r} M_{i_{1}}^{2} \cdots M_{i_{r-1}}^{2}$, which can and will be taken in $\operatorname{gr} \mathcal{Z}_{2 r}$, is a linear combination of the class sums $d_{\lambda}(2 r)$ where $\lambda \in \mathcal{E} \mathcal{P}(2 r-2)$ satisfies $|\lambda|+\ell(\lambda)=2 r-2+\ell(\lambda) \leq 2 r$, that is, $\ell(\lambda) \leq 2$. Therefore, any such $\lambda$ must be one of the following partitions

$$
\lambda^{(s)}:=(2 r-2-2 s, 2 s), \quad 0 \leq 2 s \leq r-1 .
$$

The coefficient of $d_{\lambda^{(s)}}(2 r)$ in $\sum_{i_{1}<\cdots<i_{r-1}<2 r+1} M_{i_{1}}^{2} \cdots M_{i_{r-1}}^{2}$ is $A_{\lambda^{(s)}}$. By counting the $(2 r+1)$-cycles appearing in the product $d_{\lambda(s)}(2 r) \cdot M_{2 r+1}^{2}$ and using (4.1), we calculate that the contribution from $A_{\lambda^{(s)}} d_{\lambda^{(s)}}(2 r) \cdot M_{2 r+1}^{2}$ to the coefficient $A_{2 r}$ is

$$
=\left\{\begin{array}{l}
\frac{-A_{\lambda^{(s)}} \cdot\left|D_{\lambda^{(s)}}(2 r)\right| \cdot 2\left(\begin{array}{c}
2 r-1-2 s \\
1
\end{array}\right)\left(\begin{array}{c}
2 s+1 \\
1
\end{array}\right)}{\left|D_{(2 r)}(2 r+1)\right|} \text { for } 0<2 s \leq r-1, \\
\frac{A_{(2 r-2)} \cdot\left|D_{(2 r-2)}(2 r)\right| \cdot 2\left(\begin{array}{c}
2 r-1 \\
1
\end{array}\right)}{\left|D_{(2 r)}(2 r+1)\right|} \quad \text { for } s=0,
\end{array}\right.
$$

which, by an elementary calculation, is

$$
= \begin{cases}-2 A_{\lambda(s)}, & \text { for } 0<2 s<r-1, \\ -A_{\lambda^{(s)}}, & \text { for } 2 s=r-1 \text { with } r \text { odd, } \\ 2 A_{2 r-2}, & \text { for } s=0 .\end{cases}
$$

Summarizing, we have shown that

$$
A_{2 r}= \begin{cases}2 A_{2 r-2}-A_{(r-1, r-1)}-\sum_{s=1}^{(r-3) / 2} 2 A_{(2 r-2 s-2,2 s)}, & \text { for } r \text { odd }, \\ 2 A_{2 r-2}-\sum_{s=1}^{(r-2) / 2} 2 A_{(2 r-2 s-2,2 s)}, & \text { for } r \text { even, }\end{cases}
$$

which can then be easily recast as in the lemma.

4.3 Catalan numbers and the coefficients $A_{\lambda}$

Recall the $r+1$ 'st Catalan number, $C_{r+1}$, is defined to be

$$
C_{1}=1, \quad C_{r+1}=\frac{1}{r+1}\left(\begin{array}{c}
2 r \\
r
\end{array}\right) \text {. }
$$


It is well known that a generating function for the Catalan numbers

$$
c(x)=\sum_{n=0}^{\infty} C_{n+1} x^{n+1}
$$

can be calculated to be (cf. Goulden-Jackson [3, Sect. 2.7.3])

$$
c(x)=\frac{1-\sqrt{1-4 x}}{2}=\frac{2 x}{1+\sqrt{1-4 x}}
$$

and it satisfies the quadratic equation

$$
c(x)^{2}-c(x)+x=0 .
$$

The following lemma is immediate from taking the expansion in $x$ of (4.5).

Lemma 4.4 We have the following recursive relation for the Catalan numbers:

$$
C_{2}=1, \quad C_{r+1}=2 C_{r}+\sum_{s=1}^{r-2} C_{r-s} C_{s+1} \quad(r \geq 2) .
$$

Theorem 4.5 Let $\lambda=\left(\lambda_{1}, \lambda_{2}, \ldots\right) \in \mathcal{E} \mathcal{P}$. The coefficients $A_{\lambda}$ are given by:

$$
\begin{aligned}
A_{2 r} & =-C_{r+1} \\
A_{\lambda} & =A_{\lambda_{1}} A_{\lambda_{2}} \cdots=(-1)^{\ell(\lambda)} \prod_{i \geq 1} C_{\frac{\lambda_{i}}{2}+1} .
\end{aligned}
$$

Proof By Lemma 4.2 and Lemma 4.3, we have

$$
A_{2}=-1, \quad A_{2 r}=2 A_{2 r-2}-\sum_{s=1}^{r-2} A_{2 r-2-2 s} A_{2 s} \quad(r \geq 2) .
$$

This implies that $-A_{2 r}$ satisfies the same initial condition for $r=1$ and the same recursive relation as for $C_{r+1}$ by Lemma 4.4, whence $-A_{2 r}=C_{r+1}$. The general formula for $A_{\lambda}$ follows from this and Lemma 4.2.

\section{The structures of the algebra $\mathcal{K}$ and the centers $\mathcal{Z}_{n}$}

5.1 A criterion for the subspace $\mathcal{H}^{(m)}$

Given an even positive integer $m$, let $\mathcal{H}^{(m)}$ denote the $\mathbb{B}$-submodule of $\mathcal{K}^{(m)}$ spanned by the elements $\left(d_{\lambda} d_{\mu}\right)^{*}$ where $\lambda, \mu \in \mathcal{E} \mathcal{P},|\lambda|+|\mu|=m,|\lambda|>0$, and $|\mu|>0$. 
For any partition $v=\left(2^{m_{2}}, 4^{m_{4}}, 6^{m_{6}}, \ldots\right)$ in $\mathcal{E P}$ we define the following polynomial (which lies in $\mathbb{B}$ ):

$$
\begin{aligned}
P_{v}(x) & =(-1)^{\ell(v)}\left(\begin{array}{c}
x \\
m_{2}, m_{4}, m_{6}, \ldots
\end{array}\right) \\
& =(-1)^{\ell(v)} \frac{x(x-1) \cdots\left(x-\left(m_{2}+m_{4}+m_{6}+\ldots\right)+1\right)}{m_{2} ! m_{4} ! m_{6} ! \ldots} .
\end{aligned}
$$

The proposition below is an analogue of [2, Theorem 4.3], and it can be proved as in loc. cit.. We remark that the sign in the definition of the polynomial $P_{v}(x)$, different from loc. cit., has its origin in the formula of Proposition 3.6 which is used in the proof in an essential way.

Proposition 5.1 An element $\sum_{v \in \mathcal{E} \mathcal{P}(m)} a_{\nu} d_{\nu}$ of $\mathcal{K}^{(m)}$ is contained in $\mathcal{H}^{(m)}$ if and only if

$$
\sum_{\nu \in \mathcal{E} \mathcal{P}(m)} a_{\nu} P_{\nu}(-m)=0 .
$$

5.2 The algebra generators

We shall need the Lagrange inversion formula (cf. e.g. [3, Sect. 1.2.4]) which we recall here. Let $S[[x]]$ denote the set of all formal power series in the variable $x$ with coefficients in a commutative ring $S$. Let $S[[x]]_{0}$ denote the subset of all formal power series in the variable $x$ that have zero constant term and let $S[[x]]_{1}$ denote the subset of all power series that have nonzero constant term. Let $S((x))$ denote the set of all formal Laurent series in $x$ with coefficients in $S$. Finally, given a power series or Laurent series $f$, we define $\left[x^{i}\right] f$ to be the coefficient of $x^{i}$ in the series $f$.

Lemma 5.2 Lagrange inversion formula Let $\phi(s) \in S[[s]]_{1}$. Then there exists a unique formal power series $w(x) \in S[[x]]_{0}$ such that $w=x \phi(w)$. Moreover, if $f(s) \in S((s))$, then

$$
\left[x^{n}\right] f(w)=\frac{1}{n}\left[s^{n-1}\right]\left\{f^{\prime}(s) \phi^{n}(s)\right\}, \text { for } n \neq 0 .
$$

Theorem 5.3 Let $m=2 r$ be an even positive integer. Then the coefficients $A_{\lambda}$ from (4.2) satisfy the identity

$$
\sum_{\lambda \in \mathcal{E} \mathcal{P}(m)} A_{\lambda} P_{\lambda}(-m)=2(-1)^{r} .
$$


Proof We calculate by Theorem 4.5 and the binomial theorem that

$$
\begin{aligned}
& \sum_{\lambda \in \mathcal{E} \mathcal{P}(m)} A_{\lambda} P_{\lambda}(-m) \\
& =\left[y^{m}\right] \sum_{\lambda=\left(2^{i_{2}}, 4^{i_{4}}, 6^{i_{6}}, \cdots\right)}(-1)^{\ell(\lambda)} A_{\lambda}\left(\begin{array}{c}
-m \\
i_{2}, i_{4}, i_{6}, \ldots
\end{array}\right) y^{2 i_{2}+4 i_{4}+6 i_{6}+\cdots} \\
& =\left[y^{m}\right] \sum_{N \geq 0}(-1)^{N} \sum_{N=i_{2}+i_{4}+\cdots} A_{2}^{i_{2}} A_{4}^{i_{4}} A_{6}^{i_{6}} \cdots\left(\begin{array}{c}
-m \\
i_{2}, i_{4}, i_{6}, \ldots
\end{array}\right) y^{2 i_{2}} y^{4 i_{4}} y^{6 i_{6}} \ldots \\
& =\left[y^{m}\right] \sum_{N \geq 0}(-1)^{N}\left(\begin{array}{c}
-m \\
N
\end{array}\right)\left(A_{2} y^{2}+A_{4} y^{4}+A_{6} y^{6}+\cdots\right)^{N},
\end{aligned}
$$

which, using $A_{2 r}=-C_{r+1}$ from Theorem 4.5 and (4.4-4.5), is

$$
\begin{aligned}
& =\left[y^{m}\right] \sum_{N \geq 0}(-1)^{N}\left(\begin{array}{c}
-m \\
N
\end{array}\right)\left(1+y^{-2} c\left(y^{2}\right)\right)^{N} \\
& =\left[y^{m}\right]\left(1-\left(1+y^{-2} c\left(y^{2}\right)\right)^{-m}\right. \\
& =\left[y^{m}\right]\left(1-c\left(y^{2}\right)\right)^{m} .
\end{aligned}
$$

Write $m=2 r$ and $x=y^{2}$. Now the proof of the theorem is completed by Lemma 5.4 below.

Lemma 5.4 Let $r$ be a positive integer. Then,

$$
\left[x^{r}\right](1-c(x))^{2 r}=2(-1)^{r} .
$$

Proof We rewrite (4.5) as $c(x)=x(1-c(x))^{-1}$. Applying the Lagrange inversion formula in Lemma 5.2 (by setting $w=c$ and $\phi=(1-c)^{-1}$ therein) gives us

$$
\begin{aligned}
{\left[x^{r}\right] c(x)^{k} } & =\frac{1}{r}\left[s^{r-1}\right]\left\{k s^{k-1}(1-s)^{-r}\right\} \\
& =\frac{k}{r}\left[s^{r-k}\right](1-s)^{-r}=\frac{k}{r}(-1)^{r-k}\left(\begin{array}{c}
-r \\
r-k
\end{array}\right) .
\end{aligned}
$$

Hence, by the binomial theorem and noting that $\left[x^{r}\right] c(x)^{k}=0$ for $k>r$, we have

$$
\begin{aligned}
{\left[x^{r}\right](1-c(x))^{2 r} } & =\sum_{k=1}^{r}\left(\begin{array}{c}
2 r \\
k
\end{array}\right)\left[x^{r}\right](-c(x))^{k} \\
& =\sum_{k=1}^{r}(-1)^{r} \frac{k}{r}\left(\begin{array}{c}
2 r \\
k
\end{array}\right)\left(\begin{array}{c}
-r \\
r-k
\end{array}\right) \\
& =2(-1)^{r} \sum_{k=1}^{r}\left(\begin{array}{c}
2 r-1 \\
k-1
\end{array}\right)\left(\begin{array}{c}
-r \\
r-k
\end{array}\right) \\
& =2(-1)^{r}\left(\begin{array}{c}
r-1 \\
r-1
\end{array}\right)=2(-1)^{r} .
\end{aligned}
$$


In the second last equality, we have used a standard binomial formula: for $c \geq 0$,

$$
\sum_{s=0}^{c}\left(\begin{array}{l}
a \\
s
\end{array}\right)\left(\begin{array}{c}
b \\
c-s
\end{array}\right)=\left(\begin{array}{c}
a+b \\
c
\end{array}\right) .
$$

Denote by $\mathbb{B}\left[\frac{1}{2}\right]$ the localization at 2 of the ring $\mathbb{B}$. Set $\mathcal{K}\left[\frac{1}{2}\right]=\mathbb{B}\left[\frac{1}{2}\right] \otimes_{\mathbb{B}} \mathcal{K}$ and $\mathcal{K}\left[\frac{1}{2}\right]^{(m)}=\mathbb{B}\left[\frac{1}{2}\right] \otimes_{\mathbb{B}} \mathcal{K}^{(m)}$ for $m$ even.

\section{Theorem 5.5}

1. Let $m=2 r \in \mathbb{N}$. Then, the $\mathbb{B}\left[\frac{1}{2}\right]$-module $\mathcal{K}\left[\frac{1}{2}\right]^{(m)}$ is spanned by $e_{r}^{*}$ and $\mathcal{H}^{(m)}$.

2. As a $\mathbb{B}\left[\frac{1}{2}\right]$-algebra, $\mathcal{K}\left[\frac{1}{2}\right]$ is generated by $e_{r}^{*}$ for $r \geq 1$.

Proof (1) Let $X=\sum B_{\lambda} d_{\lambda}$ be an element of $\mathcal{K}^{(m)}$, and write $\sum B_{\lambda} P_{\lambda}(-m)=q$ for some $q \in \mathbb{B}$. Recall $e_{r}^{*}=\sum_{\lambda \in \mathcal{E P}(2 r)} A_{\lambda} d_{\lambda}$. We have $\sum_{\lambda} A_{\lambda} P_{\lambda}(-m)=2(-1)^{r}$, Theorem 5.3. Then, $Y:=2 X-(-1)^{r} q e_{r}^{*} \in \mathcal{H}^{(m)}$ by Proposition 5.1, and hence $X=$ $(-1)^{r} \frac{q}{2} e_{r}^{*}+\frac{1}{2} Y$ lies in the span of $e_{r}^{*}$ and $\mathcal{H}^{(m)}$.

(2) Let $\mathcal{A}$ be the $\mathbb{B}\left[\frac{1}{2}\right]$-subalgebra of $\mathcal{K}\left[\frac{1}{2}\right]$ generated by $e_{1}^{*}, e_{2}^{*}, \ldots$ It suffices to show that $\mathcal{K} \subseteq \mathcal{A}$, or $\mathcal{K}^{(m)} \subseteq \mathcal{A}$ for all even $m \geq 0$ by induction on $m$. Certainly, $\mathcal{K}^{(0)} \subseteq \mathcal{A}$. Let $m=2 r>0$ and assume that $\mathcal{K}^{(n)} \subseteq \mathcal{A}$ for all even $n<m$. This implies that $\mathcal{H}^{(m)}$ is contained in $\mathcal{A}$ by the definition of $\mathcal{H}^{(m)}$. Since by definition $\mathcal{A}$ also contains $e_{r}^{*}$, we have by (1) that $\mathcal{K}^{(m)}$ is contained in $\mathcal{A}$.

As a corollary to Theorem 5.5, we have the following theorem by applying the surjective homomorphism $\phi_{n}: \mathcal{K}\left[\frac{1}{2}\right] \longrightarrow \mathcal{Z}\left(\mathbb{Z}\left[\frac{1}{2}\right] S_{n}^{-}\right.$) (see Proposition 3.5) and a base ring change. Another proof based on Murphy's method (cf. [9]) of Theorem 5.6 was given earlier by Brundan and Kleshchev [1] (whose working assumption that $R$ is a field of characteristic $\neq 2$ can be obviously relaxed).

Theorem 5.6 Let $R$ be a commutative ring which contains $\frac{1}{2}$ (e.g. any field of characteristic not equal to 2). Then the even center of $R S_{n}^{-}$is the $R$-algebra generated by (the top-degree terms of) the rth elementary symmetric functions in $M_{1}^{2}, \ldots, M_{n}^{2}$ with $r=1, \ldots, n$.

We remark that "the top-degree terms of" in the statement of the above Theorem is easily removable, if one follows the proof of Theorem 5.5 (1) more closely together with the surjective homomorphism $\phi_{n}$.

\section{Connections with symmetric functions}

Recall that the original results for the symmetric groups similar to our Theorem 3.4 and Proposition 3.6 were established in Farahat-Higman [2]. This led to the introduction of a ring $G$ by Macdonald (see [8, pp.131-134]), which is completely analogous 
to our current $\mathcal{F}$. Recall that $G$ is the $\mathbb{Z}$-span of $c_{\lambda}$ for $\lambda \in \mathcal{P}$, where $c_{\lambda}$ is formally the class sum of the conjugacy class in $S_{\infty}$ of modified type $\lambda$ (analogous to our $d_{\lambda}$ ). The algebra $\mathbb{Q} \otimes_{\mathbb{Z}} G$ is known to be freely generated by $c_{r}, r=1,2,3, \ldots$, where $c_{r}=c_{(r)}$. Let $\Lambda$ denote the ring of symmetric functions over $\mathbb{Z}$. Macdonald then established a ring isomorphism $\varphi: G \rightarrow \Lambda, c_{\lambda} \mapsto g_{\lambda}$, where $g_{\lambda}$ is a new basis of symmetric functions explicitly defined in [8, pp.132-134].

\section{Proposition 6.1}

(1) There exists a natural injective algebra homomorphism $\iota: \mathcal{F} \longrightarrow G$, which sends $d_{\lambda} \mapsto(-1)^{\ell(\lambda)} c_{\lambda}$ for each $\lambda \in \mathcal{E P}$.

(2) There exists an isomorphism of algebras $\psi: \mathbb{Q} \otimes_{\mathbb{Z}} \mathcal{F} \rightarrow \Lambda_{\mathbb{Q}}^{e}:=\mathbb{Q}\left[p_{2}, p_{4}, \ldots\right]$ which sends $d_{\lambda}$ to $(-1)^{\ell(\lambda)} g_{\lambda}$ for each $\lambda \in \mathcal{E P}$.

Proof (1) The multiplication in the ring $G$ is written as

$$
c_{\lambda} * c_{\mu}=\sum_{\nu \in \mathcal{P}:|v|=|\lambda|+|\mu|} k_{\lambda \mu}^{\nu} c_{\nu}, \quad \text { for } \lambda, \mu \in \mathcal{P} \text {. }
$$

By [2, Lemma 3.11] (or [8, pp.132, (5), (6)] where the notation $a_{\lambda \mu}^{v}$ was used for $\left.k_{\lambda \mu}^{\nu}\right)$, we observe that, for $\lambda \in \mathcal{E P}$ and $s$ an even integer, the constant $k_{\lambda(s)}^{\nu}=0$ unless $\nu \in \mathcal{E P}$ and $|\nu|=|\lambda|+s$. By further comparing with Proposition 3.7, we have $k_{\lambda(s)}^{v}=$ $(-1)^{\ell(\lambda)+1-\ell(\nu)} f_{\lambda(s)}^{\nu}$, which is equivalent to

$$
\iota\left(d_{\lambda} * d_{S}\right)=(-1)^{\ell(\lambda)+1} c_{\lambda} * c_{S}=\iota\left(d_{\lambda}\right) * \iota\left(d_{S}\right) .
$$

This and (3.3) imply that for $\lambda=\left(\lambda_{1}, \lambda_{2}, \ldots\right) \in \mathcal{E} \mathcal{P}$,

$$
c_{\lambda_{1}} * c_{\lambda_{2}} * \cdots=\sum_{\mu \in \mathcal{E} \mathcal{P}, \mu \unrhd \lambda}(-1)^{\ell(\lambda)+\ell(\mu)} h_{\lambda \mu} c_{\mu} .
$$

Hence, for $\mu \in \mathcal{E} \mathcal{P}$,

$$
\begin{aligned}
(-1)^{\ell(\mu)} c_{\mu} & =\sum_{\lambda \in \mathcal{E} \mathcal{P}} \tilde{h}_{\mu \lambda}\left(-c_{\lambda_{1}}\right) *\left(-c_{\lambda_{2}}\right) * \cdots \\
d_{\mu} & =\sum_{\lambda \in \mathcal{E} \mathcal{P}} \tilde{h}_{\mu \lambda} d_{\lambda_{1}} * d_{\lambda_{2}} * \cdots
\end{aligned}
$$

where $\left[\tilde{h}_{\mu \lambda}\right]$ denotes the inverse matrix of $\left[h_{\lambda \mu}\right]$. Now it follows from these identities and (6.1) that $\iota\left(d_{\lambda} * d_{\mu}\right)=\iota\left(d_{\lambda}\right) * \iota\left(d_{\mu}\right)$, i.e., $\iota$ is an algebra homomorphism.

It follows from $(1)$ and Theorem 3.9 that the image $\iota\left(\mathbb{Q} \otimes_{\mathbb{Z}} \mathcal{F}\right)$ is the polynomial algebra generated by $c_{(s)}, s=2,4,6, \ldots$. Now (2) follows by composing $\iota$ and the ring isomorphism $\varphi: G \rightarrow \Lambda, c_{\lambda} \mapsto g_{\lambda}$ and by noting that $g_{(s)}=-p_{s}$ for all (even) $s \geq 1$ (cf. [8, pp.132-134]).

Proposition 6.1 (1) is essentially equivalent to the claim that there is no cancellation in the contributions to $d_{v}$ when the (spin) permutations are multiplied between the class sums $d_{\lambda}$ and $d_{\mu}$. It fits with the computations in Example 3.3. 
Remark 6.2 In the same way as $e_{r}^{*}$ was defined right before (4.2), we can define $p_{r}^{*} \in \mathcal{F}$ as the "*-stabilization" of the $r$ th power sum of $M_{1}^{2}, M_{2}^{2}, M_{3}^{2}, \ldots$ Then one can show as in $\left[15\right.$, Propositions 5.5, 5.6] that the isomorphism $\psi: \mathbb{Q} \otimes_{\mathbb{Z}} \mathcal{F} \rightarrow \Lambda_{\mathbb{Q}}^{e}$ sends $p_{r}^{*}$ to $-p_{2 r}$. If we denote by $\eta$ the algebra isomorphism $\Lambda_{\mathbb{Q}}^{e} \rightarrow \Lambda_{\mathbb{Q}}:=\mathbb{Q}_{\mathbb{Z}} \Lambda$ sending $p_{2 r}$ to $p_{r}$ for each $r$, then the composition $\eta \psi$ sends $e_{r}^{*}$ to $(-1)^{r} h_{r}$, where $h_{r}$ denotes the $r$ th complete homogeneous symmetric function.

Problem 1 Determine explicitly the distinguished basis for $\Lambda_{\mathbb{Q}}$ which corresponds to the basis $d_{\lambda}$ in $\mathbb{Q} \otimes_{\mathbb{Z}} \mathcal{F}$ under the isomorphism $\eta \psi$.

In the original setup of symmetric groups [2,8], Macdonald's isomorphism $G \cong \Lambda$ actually identifies the $r$ th elementary symmetric function in the Jucys-Murphy elements with $(-1)^{r} h_{r} \in \Lambda$, according to [15, Theorem 5.7] (see also [10, Proposition 3.2]). So the symmetric functions in the above Problem can be viewed as another reasonable spin analogue of Macdonald's symmetric functions $g_{\lambda}$.

Acknowledgements The research of W.W. is partially supported by the NSA and NSF grants. We thank John Murray for bringing his very interesting paper to our attention when we posted an earlier version of this paper in the arXiv.

\section{References}

1. Brundan, J., Kleshchev, A.: Representation theory of symmetric groups and their double covers. In: Groups, Combinatorics \& Geometry, Durham, 2001, pp. 31-53. World Scientific, Singapore (2003)

2. Farahat, H., Higman, G.: The centres of symmetric group rings. Proc. Roy. Soc. (A) 250, 212-221 (1959)

3. Goulden, I., Jackson, D.: Combinatorial Enumeration. Series in Discrete Math. Wiley-Interscience, New York (1983)

4. Józefiak, T.: Characters of projective representations of symmetric groups. Expo. Math. 7, 193-247 (1989)

5. Józefiak, T.: Semisimple superalgebras. In: Algebra-Some Current Trends, Varna, 1986. Lect. Notes in Math., vol. 1352, pp. 96-113. Springer, Berlin (1988)

6. Jucys, A.: Symmetric polynomials and the center of the symmetric group rings. Rep. Math. Phys. 5, 107-112 (1974)

7. Kleshchev, A.: Linear and Projective Representations of Symmetric Groups. Cambridge Tracts in Mathematics, vol. 163. Cambridge University Press, Cambridge (2005)

8. Macdonald, I.G.: Symmetric Functions and Hall Polynomials, 2nd edn. Clarendon Press, Oxford (1995)

9. Murphy, G.: A new construction of Young's seminormal representation of the symmetric group. J. Algebra 69, 287-291 (1981)

10. Murray, J.: Generators for the centre of the group algebra of a symmetric group. J. Algebra 271, 725-748 (2004)

11. Nazarov, M.: Young's symmetrizers for projective representations of the symmetric group. Adv. Math. 127, 190-257 (1997)

12. Schur, I.: Über die Darstellung der symmetrischen und der alternierenden Gruppe durch gebrochene lineare Substitutionen. J. Reine Angew. Math. 139, 155-250 (1911)

13. Sergeev, A.: The Howe duality and the projective representations of symmetric groups. Represent. Theory 3, 416-434 (1999)

14. Wang, W.: The Farahat-Higman ring of wreath products and Hilbert schemes. Adv. Math. 187, 417446 (2004)

15. Wang, W.: Universal rings arising in geometry and group theory. In: Cutkosky, S.D., Edidin, D., Qin, Z., Zhang, Q. (eds.) Vector Bundles and Representation Theory. Contemp. Math., vol. 322, pp. 125140 (2003)

16. Wang, W.: Double affine Hecke algebras for the spin symmetric group. Preprint (2006). math.RT/0608074 